Revue d'histoire de l'enfance « irrégulière »

Le Temps de l'histoire

$8 \mid 2006$

Le corps du délinquant

\title{
Education morale et éducation corporelle des jeunes des classes pauvres au XIXème siècle. Entre conceptions théoriques et organisation sociale
}

\section{Christian Molaro}

\author{
CpenEdition \\ Journals \\ Édition électronique \\ URL : http://journals.openedition.org/rhei/360 \\ DOI : 10.4000/rhei.360 \\ ISBN : 978-2-7535-1646-5 \\ ISSN : $1777-540 \mathrm{X}$ \\ Éditeur \\ Presses universitaires de Rennes \\ Édition imprimée \\ Date de publication : 15 novembre 2006 \\ Pagination : 19-35 \\ ISSN : 1287-2431
}

Référence électronique

Christian Molaro, «Education morale et éducation corporelle des jeunes des classes pauvres au XIXème siècle. Entre conceptions théoriques et organisation sociale », Revue d'histoire de l'enfance " irrégulière » [En ligne], 8 | 2006, mis en ligne le 01 décembre 2008, consulté le 04 décembre 2020. URL http://journals.openedition.org/rhei/360 ; DOI : https://doi.org/10.4000/rhei.360 


\section{Éducation morale et éducation} corporelle des jeunes des classes pauvres au XIXème siècle. Entre conceptions théoriques et organisation sociale

À la fin du XVIIIème siècle et au début du XIXème siècle, émerge la conception d'un homme qui, contre la métaphysique, apparaît progressivement lié à ses actions et pensé dans ses rapports concrets au monde. Cette histoire naturelle s'affranchit des lois divines et consacre l'observation des faits. Nous pouvons certainement voir en Cabanis, en Bichat et en Gall, quelques précurseurs de cette méthode empirique. Réfutant la "séparation entre l'étude de l'homme physique et celle de l'homme moral $"{ }^{(2)}$ Pierre Cabanis, figure de proue des Idéologues, expose cette nouvelle figure de l'homme à travers une vision intégrée de l'intellect, du moral et du physique. Cette naturalisation de l'humain assujettit aussi, pour partie, ses actions à son organisation biologique primitive, et ce, dans une période qui ancre, en référence à Helvétius, les différences individuelles dans l'instruction et le milieu. Se profile dès lors la promesse d'une antinomie entre habitudes héréditaires et habitudes individuelles, antinomie qui contribuera à configurer les sciences morales et politiques de ce XIXème siècle. Ironie du sort, en 1798, Cabanis, alors député de la Seine, en appelait à la responsabilité d'un État qui se devait de secourir puis d'instruire. Il affirmait que " les principales sources de vice et des délits » participaient d'un "défaut d'instruction » de la « classe malheureuse ». Il s'agissait de combattre principalement les habitudes de paresse, et les " habitudes mendiantes $»{ }^{(3)}$ Ces recommandations, qui attestent d'une foi en la régénération morale, seront bientôt discutées en référence à cette nouvelle science médicale empreinte de philosophie, qui se propose d'éclairer un débat plus vaste : l'instruction, l'éducation et le gouvernement du plus grand nombre. Les débats et les réflexions qui accompagnent alors l'enseignement mutuel, à partir de 1815, nous paraissent réfléchir d'une façon exemplaire à la fois ces nouvelles conceptions théoriques et les enjeux liés à l'instruction des classes pauvres.

\section{Christian}

Molaro $^{(1)}$
(1) Maître de confé-

rences, université HenriPoincaré, Nancy 1, faculté du sport.

(2) Pierre Cabanis, Rapports du physique et du moral de l'homme, Paris,

Crapelet, 1805, p. X.

(3) Pierre Cabanis, Sur la nécessité de réunir en un seul système commun la législation des prisons; celle des secours publics, [au] Corps législatif, Conseil des Cinq-cents, opinion de Cabanis, député de la Seine, séance du 7 messidor, an 6, 1798, p. 3.

Christian Molaro / p. 19 à 35 
(4) Pierre Cabanis, Coup d'œil sur les révolutions et sur la réforme de la médecine, Paris, Crapelet, 1804, p. 362-363.

(5) Pierre Cabanis, Rapports du physique, op. cit., p. 486.

(6) Bally est le théoricien et défenseur de l'éducation physique dans le Journal d'éducation, organe de la Société pour l'enseignement élémentaire.

(7) Par allusion à la manière dont on enchaîne les nouveau-nés.

(8) Bally, «Éducation physique. Des divers modes d'enseignement considérés sous le rapport de la santé ", Journal d'éducation, mars 1816, p. 376 .

(9) Ibid., p. 378.

(10) Bally, «Éducation physique ", Journal d'éducation, avril 1816, p. 46.

\section{L'enseignement mutuel \\ 1.1. L'enseignement mutuel : lieu de traduction d'une nouvelle conception de l'homme}

Les propositions pédagogiques qui accompagnent l'enseignement mutuel nous paraissent traduire et prolonger ces considérations nouvelles concernant cette vision intégrée de l'individu que nous évoquions avec Cabanis. Léducation physique et l'hygiène y occupent une place centrale. Partie essentielle de la médecine, l'hygiène est aussi une partie importante de la morale. Afin de " tirer le parti le plus utile de nos facultés intellectuelles, pour diriger nos penchants et nos désirs ", il est nécessaire, écrit Cabanis, "d'approprier toutes nos habitudes physiques aux genres de nos travaux, aux dispositions morales que nous voulons cultiver en nous ".(4) L'éducation physique ne peut qu'optimiser des potentialités référées à nos dispositions organiques. Mais plus encore qu'une modification de " chaque tempérament, pris à part ", il nous invite à penser l'hygiène à l'aune de cette proposition : " considérer l'espèce humaine comme un individu dont l'éducation physique lui est confiée ». ${ }^{(5)}$

C'est à l'intérieur de telles perspectives que semble s'inscrire le docteur Bally, ${ }^{(6)}$ ardent propagandiste de l'enseignement mutuel. Il fait procès, dans le Journal d'éducation, à nombre de méthodes mortiferes qui non seulement imposent le silence au corps, mais qui souvent le mutilent. Il dénonce le recours abusif au " maillot moral ${ }^{(7)}$ dans les études, c'est-à-dire à l'application des moyens coercitifs. Cette " coutume fâcheuse de garrotter les membres " arrête "l'essor des facultés physiques ; de même qu'en exerçant une contrainte imprudente dans le cours de l'enseignement, on affaiblit les facultés morales, en même temps qu'on porte le désordre dans les organes ". ${ }^{\left({ }^{(8)}\right.}$ $\mathrm{Au}$ nombre des propositions, il préconise d'interrompre l'apprentissage " à l'instant où l'attention se fatiguerait, pour le remplacer, l'espace de quelques minutes, par de petits exercices qui, indispensables pour l'ensemble des opérations, ne laissent pas que d'égayer la jeunesse et de reposer le cerveau. Par cet innocent artifice, le corps ne se fatigue point et l'intelligence reprend toute l'énergie dont elle a besoin $"{ }^{(9)}$ Bally met ces propositions au service de la prévention des "dégénérescences " et de "l'idiotisme " auxquels peuvent conduire les méthodes trop cœrcitives. ${ }^{(10)} \mathrm{Si}$ l'on rend "à la nature ce qu'elle demande d'activité, vous prévenez ces fâcheuses dégénérescences, vous corri- 
gez même les vices de l'organisation ". ${ }^{(1)}$ C'est à ce propos qu'il nous invite à développer une éducation physique intégrée à une démarche d'éducation globale qui réconcilie "l'intellection ", le corps et la morale.

De telles propositions apparaissent ainsi susceptibles de participer à la reformulation de la question de l' "éducabilité " de l'enfant et de l'attacher " au perfectionnement général de l'espèce humaine ». En effet, " un ensemble de lois de l'économie animale portent à croire, en outre, que certaines dispositions morales se propagent également par la voie de la génération ». ${ }^{(12)}$ Mais les propagandistes de l'enseignement mutuel ne peuvent cependant se limiter au seul registre pédagogique.

\subsection{L'enseignement mutuel et l'organisation sociale}

Si l'enseignement mutuel semble alors connaître un engouement particulier, il suscite aussi de vives critiques de la part des partisans de la méthode du père de Lasalle. Cette question partage les députés et les conseils généraux. La séance de la Chambre des députés du 14 juin 1821, dont l’objet est le retranchement des $50.000 \mathrm{~F}$ affectés à l'instruction primaire, ${ }^{(13)}$ accueille aussi ce débat. La séance s'ouvre sur la discussion de ces affectations budgétaires accordées à l'enseignement élémentaire, et notamment à l'enseignement mutuel. ${ }^{(14)}$ Différents orateurs exposent en préambule les vertus et les enjeux d'un enseignement élémentaire à destination du plus grand nombre. M. Laîné, ministre, secrétaire d'état, affirme que " tous les esprits sont d'accord aujourd'hui sur le bien que produit et que peut produire dans le peuple la connaissance de la lecture et de l'écriture. Elle diminue les crimes et sert à la paix publique ». ${ }^{(15)}$ Sur " cent malfaiteurs ", le dixième seul sait lire et écrire. Le baron Pasquier, ministre des Affaires étrangères, renchérit en précisant que « le nombre des criminels est beaucoup plus considérable dans les lieux où on ne sait ni lire ni écrire ". ${ }^{(16)}$ En effet, l'ignorance rend vulnérable et peut conduire "sans crainte et sans remords aux plus effrayants excès ". ${ }^{(17)} \mathrm{Le}$ comte de Thiard, Laîné et d'autres députés, en rappelant l'esprit du préambule de cette ordonnance de Louis XVIII, en précisent les enjeux : la bonne instruction doit être adaptée à l'état des sujets et référée aux principes de la religion. Instruire le peuple contribue à l'éclairer sur le sens de son intérêt et à lui faire supporter avec résignation l'organisation sociale.
(11) Ibid., p. 46.

(12) Bally, op. cit., mars 1816, p. 372. Théorie qui alimentera la métaphore de la contagion. Outre chez Cabanis, nous retrouvons déjà cette idée chez Condorcet.

(13) Somme suspectée d'être au seul profit de l'enseignement mutuel. Précisons que plus de 2 millions de francs sont alors affectés aux collèges royaux.

(14) À l'ordre du jour de la séance, figurent deux amendements : le premier concerne la non reconduction de l'affectation des 50.000 francs destinés à l'instruction primaire et le second propose que cette somme ne soit allouée qu'aux frères de la doctrine chrétienne. Ces deux amendements seront rejetés. Discussion sur l'enseignement mutuel à la Chambre des députés, séance de la Chambre des 
députés du 14 juin 1821 ,

Paris, L. Colas, imprimeur libraire, 1821.

(15) Ibid., p. 39.

(16) Ibid., p. 39

(17) Ibid., p. 39.

(18) Chevalier Cadet de Gassicourt, «De la nécessité d'introduire l'enseignement élémentaire dans les maisons de détention ", Journal d'éducation, décembre 1815 , p. 200-204.

(19) « Extrait d'un rapport fait au Conseil général des prisons par la commission nommée pour l'instruction primaire ", Journal d'éducation, IX, octobre 1819, p. 57-69, p. 60.

(20) Comte Alexandre de Laborde, « Docteur Bell, M. Lancaster, Plan d'éducation pour les enfants pauvres ", Journal d'éducation, novembre 1815, I, p. 119-127, p. 125.
Le Journal d'éducation s'est attaché, depuis quelques années, à répondre à ces préoccupations. La méthode constitue une première réponse. Dénonçant l'oisiveté, frère d'arme de l'ignorance, promis à une belle postérité, le chevalier Cadet de Gassicourt, militant pour l'introduction de l'instruction dans les maisons de détention, disserte sur le vagabondage des enfants à Paris et sur leurs mœurs en ces termes : «Voici donc, Messieurs, une pépinière de scélérats que le châtiment même prépare à de plus grands désordres. L'ignorance et l'oisiveté sont les deux causes de si grands maux, et votre institution peut y remédier. " ${ }^{(18)}$ Il faut donc présider à leur emploi du temps, diriger leur instruction afin de garantir un avenir qui passe par la régénération morale. Le Journal d'éducation d'octobre 1819 reproduit les extraits d'un rapport adressé au Conseil général des prisons, rapport dans lequel sont vantés les bienfaits d'une instruction élémentaire à même de réformer les inclinaisons vicieuses des enfants. L'enseignement mutuel y est présenté par le duc de Broglie, le comte de Laborde, le comte de Saint-Aulaire... comme la méthode la plus intéressante. Il existe, nous disent les rapporteurs, trois méthodes d'enseignement élémentaire : l'enseignement individuel, l'enseignement simultané et l'enseignement mutuel. Le premier, " qui consiste à faire répéter chaque élève séparément, n'est guère applicable à une école nombreuse ou à une instruction rapide ", la méthode de l'enseignement simultané, " qui partage l'école en plusieurs subdivisions, est plus parfaite, mais elle occasionne encore la perte de beaucoup de temps, parce qu'elle force toutes les subdivisions à rester oisives lorsqu'on fait répéter l'une d'elles, et la perte de temps dans une prison ne peut qu'être préjudiciable ». Seule la dernière méthode, l'enseignement mutuel, "éloigne tous ces obstacles ». ${ }^{(19)}$ Mais les propagandistes de l'enseignement ne s'arrêtent pas à ce seul aspect de la méthode, il s'agit aussi de donner l'orientation politique et morale de ces propositions.

L'instruction doit être au service de la reconduction de l'organisation sociale. Elle ne doit pas susciter le désir de s'émanciper de sa condition et il faut le rappeler. L'ignorance comme l'instruction peuvent également conduire au désordre. Le choix reste difficile entre le barbare et le pauvre émancipé. Mais, pour le comte de Laborde, " on se trompe en disant que l'instruction est dangereuse ou inutile dans les classes inférieures de la société ! " (20) Pour ce défenseur de l'enseignement mutuel, inscrire de telles visées dans un pro- 
jet d'éducation plus vaste, qui invite à l'accomplissement de devoirs qui sont des devoirs de chrétien, parait nécessaire. Il invoque le secours de la religion et en appelle à une nature et une éducation qui feraient système. La nature n'a donné à "l'homme de la terre [que] des forces pour ce pénible sort ", ${ }^{(21)}$ l'éducation seule lui fournira le courage. Il faut réussir le passage d'un asservissement à un assujettissement volontaire - assujettissement éclairé - duquel le sujet tirerait divers bénéfices, dans le plus grand intérêt du corps social.

"Au lieu de la patience servile qui le soutenait à peine en l'abrutissant, la vertu l'élève et le console. Alors il peut pousser avec fierté la charrue qui l'honore, et recevoir avec douceur la tâche que le ciel lui impose. Il ne cherchera point une meilleure destinée hors du cercle de ses devoirs, car la meilleure destinée est pour lui dans l'accomplissement de ses devoirs. " (22)

Cet enseignement mutuel affirme ainsi sa participation au maintien de l'ordre social, parfois son allégeance au religieux, tout en exprimant, dans ses propositions pédagogiques, une vision plus émancipée, non dualiste de l'homme. Ces propositions pédagogiques, qui concilient le physique et le moral, sont assujetties à cette conviction que des habitudes d'ordre et que l'activité de l'enfant peuvent faire obstacle aux dégénérescences et aux inclinaisons vicieuses, et à ce titre s'adresser aussi au monde des prisons. Elles alimentent à leur manière le débat sur la possible correction des dispositions vicieuses de la jeunesse. Dans cette première moitié du XIXème siècle, notamment à partir des années 1930, de telles considérations, associées à cette foi en la régénération morale, vont apporter leur contribution, plus encore qu'à l'enseignement élémentaire, à la redéfinition de l'éducation correctionnelle de l'enfance coupable.

\section{La question de la régénération morale}

\subsection{L'affirmation d'une foi en la régénération morale}

En 1830, le comte René de Bouillé mobilise les mêmes arguments quand il fait l'éloge de la prison pénitentiaire de Genève. Combiner le "régime physique ", la " direction morale ", soumettre les détenus à " une vie régulière et laborieuse " afin qu'ils contractent " une habitude d'occupation et de bonne conduite ", sont autant de clés de réussite et de possible "régénération ". ${ }^{(23)}$ Benjamin Appert partage les mêmes convictions et s'attache à les diffuser : « Le
(21) Ibid., p. 126.

(22) Ibid., p. 126.

(23) Comte René de Bouillé, "Visite à la prison pénitentiaire de Genève ", Revue des deux mondes, juillet 1830 , p. 7-19. Cette prison, qui sépare les moins de 16 ans des autres détenus, impose le plus grand silence et le son d'une cloche rythme les activités. 
(24) Benjamin Appert,

Bagnes, prisons et criminels, quatrième partie,

Mélanges, Paris, Guilbert, 1836, p. 230. Il relate

l'expérience de la prison des Madelonettes où, pour briser « l'esprit de corps " des détenus et à l'instar de l'enseignement mutuel qui s'appuyait sur des élèves relais, les détenus cette fois-ci « sont embrigadés sous des caporaux et des sergents, toujours choisis parmi les meilleurs sujets " (p. 250).

(25) Ibid., p. 233.

(26) M. F. du Cellier, Histoire des classes laborieuses en France, Paris, Didier et Cie, 1859, p. 410 .

(27) Benjamin Appert, op. cit., p. 233.
(28) Aubert de Vitry,

«Compte général de la justice criminelle en France de l'administration pendant l'année 1834 ", Revue mensuelle d'économie politique, 1836, tome V, p. $69-385$, p. 377.

(29) Jacques Bourquin, «Un statut qui précède le métier ", le Temps de l'histoire, $\mathrm{n}^{\circ} 1,1998$, p. 57-66.

(30) Jules Simon,

" L'apprentissage des jeunes ouvriers dans la petite industrie ", Revue des deux mondes, tome 55, 1865, p. 717-742. temps n'est pas loin où les plus incrédules seront convaincus de la possibilité de cette régénération morale, [...] il n'est pas de vicieux penchant, si invétéré qu'il soit, qui ne cède tôt ou tard à des habitudes de travail, d'ordre et de subordination [...] à l'influence des bonnes doctrines, aidées de l'encouragement des bons exemples ", ${ }^{(24)}$ écrit-il. Il insiste aussi sur la propreté corporelle, rappelant « combien l'ordre physique a de puissance et combien il influe sur le sentiment moral ". ${ }^{(25)}$ Les colonies agricoles, et tout particulièrement la colonie de Mettray, objectivent aussi de cette conviction que les bons sentiments atrophiés par suite d'un défaut d'éducation peuvent être facilement réveillés, aussi bien chez l'enfant du pauvre que chez l'enfant du riche, et s'imposer " aux mauvais instincts ". (26) L'instruction semble cependant quelque peu oubliée au profit de l'éducation morale, même si Appert fait état d'un enseignement élémentaire d'une heure et demi dans le centre. Temps que l'auteur semble juger insuffisant, mais " le traité avec l'entreprise générale des travaux " ${ }^{(27)}$ ne permet pas plus. Par ailleurs, les statistiques ne tarderont pas à conforter les partisans de l'éducation dans leur conviction qu'il y a plus à éduquer qu’à instruire. Dans son "Compte général de la justice criminelle pour l'année 1834 ", Aubert de Vitry, s'appuyant sur Adolphe Quételet pour la période de 1828 à 1831, fait état d'une corrélation entre le crime contre les personnes et le développement de l'intelligence et de l'instruction. De même, "les départements les plus pauvres de la France, et en même temps les moins instruits, tels que ceux de l'Indre, du Cher, de la Haute-Vienne, de l'Allier, etc., sont "en même temps les plus moraux ». ${ }^{(28)}$ L'instruction n'est pas l'éducation.

Cependant une inflexion va marquer la seconde moitié du XIXème siècle. L'évolution des colonies agri- 
coles, par exemple, en témoigne. Pour Jacques Bourquin, le travail perd peu à peu sa fonction d'éducation, de moralisation et nous nous dirigeons progressivement vers une "logique de garde, de discipline " et " de châtiment ». ${ }^{(29)}$ Il faudrait certainement mettre " au crédit » de cette évolution la difficile reconnaissance de l'enfance comme catégorie sociologique et juridique dans ce XIXème siècle. En atteste, par exemple, la laborieuse avancée de la législation du travail les concernant. Évoquons rapidement la loi de 1841, votée difficilement. Cette loi pénalise les fabricants, invoquait-on comme argument. Jules Simon, plus de vingt ans après, regrette que cette loi n'ait pas encore une portée générale, qu'elle ne concerne que la grande industrie. De même déplore-t-il l'absence d'un " corps d'inspecteurs salariés » ${ }^{(30)}$ qui rendrait effective ces avancées, qui en garantirait l'application réelle. Dans un article précédent, il déplorait l'introduction massive des femmes et des enfants dans les ateliers, introduction qui tendait à " modifier gravement la vie de famille, sinon à l'anéantir ", de même que l'altération subséquente de la santé, ainsi que "la privation de toute instruction et de toute éducation ». ${ }^{(31)}$ Les industriels ne sont que peu enclins à se passer de cette main d'œuvre enfantine bon marché et malléable. Comme l'écrit alors Édouard Ducpétiaux, ${ }^{(32)}$ pour la plupart des fabricants, "la dépendance absolue du travailleur est un fait qu'il importe de renforcer au lieu de la détruire » et " l'extension de l'instruction, loin d'être utile, aurait au contraire l'inconvénient d'éveiller chez le peuple des idées, des besoins qu'il ignore et qu'il fait bien d'ignorer ».. ${ }^{(33)}$

D'une façon concomitante, l'évolution de conceptions théoriques, en accordant un poids plus important au déterminisme biologique, ${ }^{(34)}$ va participer à ébranler davantage encore cette idée de la rédemption par l'éducation ou par le travail.

\subsection{L'évolution de la science médicale : la régénération morale en question}

En début de siècle, les déterminations internes et externes cohabitent, sans qu'il soit toujours possible de faire la part de ce qui revient aux dispositions et à l'influence du milieu. Un des pères de la psychiatrie française, Philippe Pinel, écrit que l'éducation des enfants peut être dirigée "à contre sens, et ses effets se combiner si bien avec une faiblesse originaire de l'entendement ",
(31) Jules Simon,

"L'ouvrier de huit ans",

Revue des deux mondes, tome 54,1864 , p. $715-$ 735 , p. 716.

(32) Inspecteur général des prisons et des établissements de bienfaisance en Belgique.

(33) Édouard

Ducpétiaux, De la condition physique des jeunes ouvriers et des moyens de l'améliorer, tome II, Bruxelles, Méline, Cans \& Cie, 1843, p. 227.

(34) Nous renvoyons notamment à différentes contributions de l'ouvrage collectif : Laurent Mucchielli, [dir.], Histoire de la criminologie française, Paris, L'Harmattan, 1994. 
(35) Philippe Pinel, Traité médico-philosophique sur l'aliénation mentale, Paris, J. A. Brosson, 1809, p. 17.

(36) Ibid., p. 17.

(37) Ibid., p. 18.

(38) Franz Joseph Gall et Johann Gaspar Spurzheim, Anatomie et physiologie du système nerveux en général et $d u$ cerveau en particulier, tome IV, Paris, Schoell, 1810 , p. 40 sqq.

(39) Appert a présidé à plusieurs reprises la société de phrénologie de Paris.

(40) Benjamin Appert, op. cit., p. 298.

(41) Ibid., p. 300.

(42) Disciple d'Esquirol, lui-même disciple de Pinel.

(43) Jacques-Joseph Moreau, La psychologie morbide dans ses rapports avec la philosophie de qu'il demeure « du doute sur ce que l'on doit attribuer à l'une de ces causes plutôt qu'à l'autre ». ${ }^{(35)}$ Prenant l'exemple de deux frères mineurs dont l'entendement " resta sans se développer ", il avoue, en évoquant leur éducation et « leur corps affaibli », ne pas savoir ce qui relève " du vice d'une institution pareille » ou d'une « disposition primitive ». ${ }^{(36)}$ Il n'écarte nullement les déterminations externes comme explication des comportements répréhensibles des enfants et fait état de nombre de cas où les menaces et les coups peuvent produire " des penchants pervers » ou précipiter " dans une aliénation déclarée ». ${ }^{(37)}$ Des auteurs comme Franz Joseph Gall et Johann Gaspar Spurzheim $(1810,1811)^{(38)}$ apporteront leur contribution à ce débat. Si la phrénologie de Gall ne suscite initialement que quelque curiosité, voire que quelque amusement, les années 1830, pour Appert, ${ }^{\left({ }^{(3)}\right)}$ la réhabilitent. Si elle reste contestée, elle alimente cependant la réflexion d'une législation criminelle qui « se questionne " quant à l'appréciation des délits et quant au statut du criminel. Il s'agit de se poser la question de la probabilité du crime. Les clés résident peut-être dans l'organisation biologique primitive et notamment dans le cerveau. "L'examen du cerveau chez les coupables, depuis l'assassin jusqu'au voleur le moins marquant, finirait peut-être par prouver qu'il existait chez les hommes une tendance anticipée vers tel ou tel crime. " ${ }^{(40)}$ Appert cite le procureur général Dupin aîné qui, en 1833, rend compte de l'existence d'une sensibilité qui poserait le crime comme prolongement « d'une affection cérébrale ». ${ }^{(41)}$ Ce courant philanthropique, alimenté par la phrénologie, en appelle à confier les coupables aux hôpitaux, au gymnase et aux écoles, Dupin aîné en appelle quant à lui à la prudence. Ces idées se développeront dès lors chez nombre d'auteurs - Jacques-Joseph Moreau ${ }^{(42)}$ (1845, 1859), Bénédict-Auguste Morel (1857, 1860), Cesare Lombroso (1876...), Paul Moreau (1882, 1888), Henry Maudsley (1888), Charles Féré (1888...), Paul Aubry (1894), Émile Laurent (1908)... -, auteurs qui affirmeront, avec leurs différences, le poids de l'empreinte héréditaire.

L'aliéniste Jacques-Joseph Moreau, dans son ouvrage de 1859, conteste la toute puissance de l'éducation, idée référée à Locke et à Helvétius. Sans nier "l'influence de l'éducation sur l'intelligence proprement dite et sur les facultés affectives et morales ", il leur oppose "l'activité native de ces facultés ". ${ }^{(43)}$ Cette thèse s'affirme davantage encore dans les années suivantes. Chez Paul 
Moreau, si nous retrouvons les deux catégories de causes, les causes « subjectives ", qui tiennent à la nature morale, sont posées comme "prédisposantes » et sont sans contredit les plus importantes. ${ }^{(44)}$ "L'organisme est tout. " ${ }^{(45)}$ Les actes répréhensibles, perçus trop longtemps comme des défauts d'éducation ou résultant "d'exemples journaliers ", comme "des instincts fâcheux que l'on doit corriger et punir ", ne sont que des "symptômes de maladie qu'il faut traiter physiquement et moralement ${ }^{(46)}$ Pour Paul Moreau, l'explication des faits monstrueux auxquels peuvent se livrer les enfants est à rechercher dans les antécédents héréditaires, dans les lois fatales de la dégénérescence. Dès lors, l'amendement des criminels de nature ou d'habitude devient difficile. La continuité entre l'homme, l'animal et les races inférieures peutêtre établie. Dans L'histoire de la folie, Michel Foucault nous renvoie, avec Étienne Esquirol, à l'image de la bête compagnon naturel de la folie, aux menaces les plus sourdes qui pèsent sur la civilisation et l'ordre social. Morel, quant à lui, établit cette correspondance entre l'aliénation mentale, la dégénérescence et le salut de la race. Cette dégénérescence ${ }^{(47)}$ est certainement un danger plus grand " pour les sociétés modernes que ne l'étaient pour les sociétés anciennes l'invasion des barbares ". ${ }^{(48)}$ En effet, selon cette théorie, la pathologie physique d'un parent - tuberculose, rachitisme... - peut affecter l'enfant au-delà de la seule tare physique, puisque est transmis ce que l'on appelle un "quantum de dégénérescence ». Un rachitique, un tuberculeux... peut donner naissance à un alcoolique, à un criminel. Dans cette seconde moitié du XIXème, l'hérédité alimente donc l'étiologie des comportements délinquants et une figure de l'anthropologie française comme Charles Letourneau accrédite, à sa façon, cette théorie : "Quand certaines empreintes nerveuses sont suffisamment fixées, incarcérées, organisées dans les centres nerveux, elles deviennent héréditaires. Spécialement en ce qui concerne la moralité, on peut dire que chaque individu déprave ou moralise sa postérité, comme il a été moralisé ou dépravé par ses ancêtres. " ${ }^{(49)}$ Et ce qui accompagne cette prégnance de l'hérédité directe - ou transformée -, c'est aussi l'affirmation d'une hypothèse anthropologique qui pose l'homme comme naissant avec un penchant naturel à la méchanceté, voire "à la férocité ", ${ }^{(50)}$ imposant de fait une certaine figure de l'enfance. l'histoire ou De l'influence des névropathes sur la dynamisme intellectuel, Paris, Masson, 1859, p. 9. [Voir aussi la section II de l'ouvrage].

(44) Paul Moreau (de Tours), De l'homicide commis par les enfants, Paris, Asselin et Cie, 1882, p. 20.

(45) Ibid., p. 20.

(46) Paul Moreau (de Tours), La folie chez les enfants, Paris,

J. B. Baillière, 1888, p. 2.

(47) Dégénérescence qui semble, pour Morel, plus participer de la déviation que d'une résurgence de l'atavisme.

(48) Bénédict-Augustin Morel, Traité des maladies mentales, Paris, Masson, 1860 , p. 660.

(49) Charles Letourneau, L'évolution de la morale, Paris, A. Delahaye, 1887 , p. 31 . Il est préfa- 
cier d'une réédition de

L'homme criminel (1887).

Il y prend alors quelques

distances avec les thèses de

Cesare Lombroso.

(50) Paul Moreau (de Tours), De l'homicide..., op. cit., p. 10.

(51) Ibid., p. 8.

(52) Ibid., p. 6.

(53) Émile Durkheim,

Education et sociologie,

Paris, PUF, 1992, p. 52.

Dans d'autres textes, il considère par ailleurs cette question comme un faux problème. Voir Émile

Durkheim, L'éducation morale, Paris, Félix Alcan, 1925, huitième leçon, p. 167.

(54) Alfred Fouillée, «Les jeunes criminels, l'école et la presse ", Revue des deux mondes, tome CXXXIX, 1897, p. 417-449, p. 423.

(55) Ibid., p. 423.

\section{La figure de l'enfance}

\subsection{Un enfant gouverné par l'instinct et les passions}

Paul Moreau s'appuie sur Gall, Broussais... pour asseoir cette "innéité de la tendance au crime ». ${ }^{(51)}$ En général, l'enfant " préfere le mal au bien parce qu'il satisfait davantage sa vanité et qu'il y trouve plus d'émotion ». ${ }^{(52)}$ Une telle représentation de l'enfance va s'imposer dans cette fin de siècle. Un auteur comme Émile Durkheim, qui relativisera ces " vagues et incertaines tendances qui peuvent être dues à l'hérédité », atténuera cette représentation tout en continuant à présenter cet enfant comme naturellement " asocial » et " égoïste ». ${ }^{(53)}$ Pour l'heure, et pour Alfred Fouillée, " ce qui est certain, c'est que les instincts mauvais et même criminels sont fréquents chez les enfants ". ${ }^{(54)}$ Fouillée évoque également une représentation qui a cours : "On a dit que ces petits sauvages [...] volontiers menteurs, cruels et égoïstes " reproduisent dans leur développement « les phases de l'espèce humaine ". ${ }^{(55)}$ Et, même si des voix s'élèvent régulièrement pour rappeler que les vices sont également distribués, pour affirmer que « la disposition à la paresse, à la violence, à l'improbité » n'est pas "plus commune dans une classe que dans l'autre ", ${ }^{(56)}$ ces "petits sauvages " ne manquent pas de renvoyer aux classes populaires. Force est de constater, écrit Cellier, que c'est à " la classe laborieuse qu'appartient la presque totalité des enfants dont les actes tombent sous la répression juridique $» .{ }^{(57)}$ Ce que confirment les statistiques et les travaux des aliénistes.

\subsection{Un enfant pauvre soumis à une double contagion}

Ainsi le docteur Paul Moreau nous propose-t-il, remaniant la classification de Félix Voisin, ${ }^{(58)}$ trois classes d'enfants :

- les enfants ayant des ascendants atteints "d'affections ou d'anomalies nerveuses ";

- les enfants normaux, mais dont une mauvaise éducation « a fait prendre une direction vicieuse ";

- les « enfants nés extraordinairement », qui «forment les grands hommes ou les grands scélérats" selon l'influence du milieu. ${ }^{(59)}$

Cette première catégorie d'enfants, marqués par des " prédispositions organiques vicieuses » transmises, " appartient aux classes pauvres ». ${ }^{(60)}$ Ces derniers "sont généralement nés d'alcoolisés, de voleurs, de criminels, de 
gens perdus de vices et de débauches, tristes individus qui dans les grandes villes vivent presque toujours en communauté, constituant un véritable État dans l'État ". ${ }^{(61)}$ Ces classes vivent en marge, cumulent l'hérédité morbide et l'habitude et "propagent une population criminelle [...] ne se mêlant qu'entre eux, ils ne donnent naissance qu'à une classe d'êtres dépravés, héréditairement portés au crime. Leur maladie morale existe $a b$ ovo, et bientôt l'habitude devient une seconde nature qui s'ajoute à leur dépravation morale originaire ». ${ }^{(62)}$ S'affirme, à travers cette classification, une catégorie d'enfants non amendables, incurables : il s'agit majoritairement des enfants qui appartiennent aux classes pauvres. La première catégorie est victime de l'hérédité, quant à la deuxième, elle succombera à la contagion par l'exemple. En effet, la partie saine n'est pas épargnée, puisque facilement influençable. Honoré Antoine Frégier, en 1840, relatait déjà cette influence des criminels qui débauchent ces honnêtes travailleurs. "Ces malheureux qui, par l'exercice de leur profession, se rattachaient encore en apparence à la masse des ouvriers honnêtes et laborieux, dépouillent peu à peu, sous la maligne influence de leurs compagnons de désordres, les habitudes de travail qui leur restaient et finissent par embrasser leur vie fainéante et criminelle. " ${ }^{(63)}$ En fin de siècle, rien n'a changé. Cet homme honnête des classes populaires est vulnérable de par sa nature, puisque « l'être le plus vertueux renferme un criminel ensommeillé : il suffit d'un éclair parti de l'ambiance pour séparer du moi, jusqu'alors impeccable, le moi violateur, homicide ou incendiaire ". ${ }^{(64)} \mathrm{Il}$ y a, en quelque sorte, enfermement dans une double contagion : une contagion par l'hérédité et une contagion par l'exemple. Quand le vice n'est pas associé à la perversité ou à une hérédité morbide, il se propage par imitation, et l'enfant est tout particulièrement concerné, puisqu'il est naturellement imitateur. ${ }^{(65)}$ Pour Gabriel d'Haussonville, " la gangrène morale gagne vite et facilement ces jeunes êtres ». ${ }^{(6)}$ Force est de constater que l'enfant des classes pauvres se trouve enfermé dans un cycle vicieux. Qui plus est, la laideur, la faiblesse du corps, sa stérilité, les stigmates... qui l'affectent sont autant de marques visibles de la dégénérescence et du crime, et celles-ci n’épargnent pas l'enfant.

Le roman social, sous la Monarchie de Juillet, a participé à imposer cette figure d'un être déchu qui conjugue la régression physique et l'insensibilité morale. Un certain discours scientifique, dont les représentants les plus

(56) M. F. du Cellier, op. cit., p. 409.

(57) Ibid., p. 409.

(58) Félix Voisin, Des causes morales et physiques des maladies mentales et de quelques autres affections nerveuses telles que l'hystérie, la nymphomanie et le satyriasis, Paris,

J. B. Baillière, 1826. Les quatre classes d'enfants de Voisin étaient : les " normaux ", les « extraordinaires" (donnant les grands hommes et les grands scélérats), les "pauvres d'esprit » et les "aliénés ». Moreau réunit les deux dernières classes.

(59) Paul Moreau, De l'homicide..., op. cit., p. 20.

(60) Ibid., p. 11.

(61) Ibid., p. 11.

(62) Ibid., p. 11.

(63) Honoré Antoine Frégier 1840, in Laurent Mucchielli, op. cit., p. 12. 
(64) Armand Corre, [dir], in préface à Paul Aubry, La contagion $d u$ meurtre, Paris, Félix Alcan, 1894 , p. XXI.

(65) Édouard Ducpétiaux, op. cit., p. 200.

(66) Gabriel d'Haussonville, « L'enfance à Paris. VI. Les rendez-vous du crime, les jeunes adultes et l'éducation correctionnelle ", Revue des deux mondes, tome XXXI, 1879 , p. 346-374, p. 347.

(67) Ibid., p. 348.

(68) Ibid., p. 346.

(69) Voir le classique Louis Chevalier, Classes laborieuses et classes dangereuses à Paris, pendant la première moitié $d u$ XIXème siècle, Paris,

Hachette, 1984.

(70) Charles Féré,

Dégénérescence et criminalité. Essai physiologique, Paris, Félix Alcan, 1888, p. 101. entendus dans ces années 1880 appartiennent à l'école anthropologique italienne (emmenée par Lombroso), apportera son crédit à cette représentation d'êtres " mal constitués » et incapables de s'adapter à notre milieu social. Le corps est devenu à la fois le lieu de détermination et de déposition de la preuve, de la dégénérescence et de la perversité. Ces propositions seront bien sûr discutées, contestées. Mais il est des descriptions qui, apparemment empreintes de sympathie, se révèlent insidieuses quand elles nous invitent à dépasser cette première compassion pour arrêter enfin notre regard sur autant d'objectivations de ce second type de contagion que nous avons évoqué. Cela concerne l'hexis corporelle, les multiples façons de s'habiller et de parler, cela renvoie aux attitudes... : «On est étonné de voir tant de visages imberbes, sur lesquels le vice et la débauche ont cependant imprimé déjà leur souillure ineffaçable " ${ }^{(67)}$ écrit Haussonville. Les regards fuient, se détournent... Haussonville évoque encore un dessin de Daumier dans lequel trois juges interrogent " une petite fille en haillons ", petite fille qui, " la tête baissée, le coin de son tablier ramené dans sa main, tourne vers le spectateur une physionomie sournoise et hypocrite ". ${ }^{(68)}$ Se prévalant de son expérience des tribunaux, au nom du devoir de franchise et d'honnêteté et après avoir exprimé sa compassion, Haussonville apporte toute sa caution à cette représentation.

Ainsi le crime et le délit ne concernent-ils pas tant la victime que le corps social dans son ensemble, voire la race. Dans cette seconde moitié du XIXème, derrière le délit d'un jeune délinquant - assimilé au milieu populaire,$-{ }^{(69)}$ nous voyons de plus en plus le dégénéré, c'est-à-dire la menace potentielle qu'il représente pour la partie saine du corps social. Comment dès lors penser les délits mineurs comme des délits mineurs, puisqu'ils sont signes de dégénérescence et annonciateurs de menaces bien plus graves. La mise en perspective statistique et/ou la mise en intrigue du fait divers ne peuvent pas être dissociées de cet arrière-plan. Cela constitue un des référentiels forts de cette seconde moitié du XIXème et ce référentiel pèse certainement pour partie sur les pratiques qui alimentent un mode distributif-répressif. Ces théories de la dégénérescence posent une menace qui excède le seul présent. Protéger ne suffit plus, il faut prévenir car, " en matière de dégénérescence, l'on peut dire que le présent prépare l'avenir ». ${ }^{(70)}$ Mais les dernières années du siècle vont relativiser ces théories. 


\section{La fin du XIXème siècle}

\subsection{Le retour de l'éducatif}

Un spiritualisme positiviste et une certaine philosophie bientôt relayés, dans cette fin de siècle, par une sociologie criminelle emmenée par L’Année sociologique, objecteront de plus en plus à cette hérédité la " part sociale » de l'individu ${ }^{(71)}$ ou du milieu. Un philosophe comme Jean-Marie Guyau oppose à "l'habitude héréditaire ou ancestrale " l'habitude individuelle. S'appuyant sur les évolutions de la psychophysiologie et sur les récentes avancées de la "suggestion ", ces diverses contributions entendent réhabiliter l'éducation. Est affirmé comme possible le fait de « créer dans un esprit, à tout moment de son évolution, un instinct artificiel capable de faire équilibre plus ou moins longtemps aux tendances préexistantes ", ${ }^{(72)}$ c'est-à-dire aux instincts héréditaires. Mais cette théorie va se voir opposer la force de l'exemple. A l'influence de la suggestion par l'idée, on objecte l'influence de la contrainte imitative par l'acte. Les leçons de choses offertes sous forme de récit ne résisteraient pas à la force de l'exemple : "C'est par l'acte, bien plus que par l'idée, ou par l'acte associé à l'idée que l'imitation recrute avec le plus d'avantages pour l'armée de la corruption et du délit. » ${ }^{(73)}$ Néanmoins le débat apparaît à nouveau plus équilibré, renouant avec la mesure de la première partie du XIXème siècle. Si le congrès d'anthropologie criminelle de Genève (1896) consacre l'opposition de deux " grandes écoles scientifiques » qui ancrent les racines du crime dans l'organisme individuel, pour l'une, dans le milieu social, pour l'autre, de plus en plus d'auteurs tentent de dépasser cette opposition. Signalons aussi que la plupart des contributions d'une revue comme L'Année sociologique s'attachent, à partir de 1896-1897, à montrer que l'origine de la criminalité infantile est sociale et non atavique et que le régime répressif doit être compté au nombre des facteurs sociaux de cette criminalité.

Encore une fois, cette inflexion des doctrines apparaît consubstantiellement liée à une inflexion dans la prise en compte des jeunes délinquants. Le contexte est marqué par des avancées législatives : lois de 1889 et de $1898 .{ }^{(74)}$ Le regard que les professionnels portent sur l'enfance coupable et sur son « éducation correctionnelle " change progressivement. Albanel se demande s'il ne faut pas " trouver des équivalents et des substituts à la peine ». S’il trouve cette " théorie plutôt philosophique » et " un peu excessive en ce qui concerne l'adulte ", 
(75) Louis Albanel, Le crime dans la famille, Paris, J. Rueff, 1900, p. 204.

(76) Henri Thulié, Le dressage des jeunes dégénérés ou orthophrénopédie, Paris, Félix Alcan, 1900, p. 251.

(77) Ibid., p. 308.

(78) Hector Depasse, " De la quantité d'instruction ", La Nouvelle Revue, 1896 , tome 98 , p. 260270, p. 261.

(79) Alfred Fouillée, op. cit., p. 424. elle " doit être acceptée complètement vis-à-vis des mineurs ", ${ }^{(75)}$ écrit-il. Ce qu'objective aussi cette proposition d'Albanel, c'est à la fois la difficulté et le devoir de réaliser cette distinction entre l'enfance et le monde adulte. Henri Thulié voit également, dans cette période, se redessiner la volonté de remplacer la punition par une « éducation réformatrice ». ${ }^{(7)}$ La société " pour se protéger d'une façon efficace, ne doit pas se borner à les isoler dans des établissements de répression [...] parce qu’il y revient plus dangereux. [...] La société doit s'efforcer pendant que le dégénéré est jeune et par conséquent transformable, de le transformer ». ${ }^{(77)}$ Se pose à nouveau la question de la méthode. Comment transformer ces jeunes?

La fin du XIXème et le début du XXème siècle semblent donc renouer avec des considérations éducatives qui étaient pour partie présentes dans les premières décennies du siècle..., à l'exception peut-être de cette vision d'une éducation corporelle intégrée dans une éducation globale. C'est en tout cas dans ce contexte que l'on commence aussi à réhabiliter l'école.

\section{2. Éducation corporelle, morale et patriotique pour les jeunes des classes populaires}

La défiance envers une instruction potentiellement corrélée au crime et au désordre reste bien présente en fin de siècle. Maurice Barrès, Charles Maurras, Léon Daudet... associeront la recrudescence des crimes et délits des mineurs à l'obligation de la scolarité. Bien sûr, certains dénonceront cette " classe d'esprits assez infatués de leur propre instruction ", qui recommence à brandir "ce que l'on n'entendait plus depuis une vingtaine d'années » - cette menace que ferait courir à la société une instruction distribuée sans mesure. ${ }^{(78)}$ Mais le contexte, alimenté par force statistiques affirmant la forte augmentation de la criminalité juvénile, contribue à donner corps à cette idée. Des auteurs comme Lapie, Brunetière, Fouillée examinent cette question de la corrélation de l'école et de la criminalité. Alfred Fouillée nous fait remarquer que cette dernière augmente alors que l'instruction est obligatoire, mais il conclut " que la relation entre ces deux faits reste une inconnue ${ }^{(79)}$ Gaston Richard, quant à lui, se félicite des études statistiques que mène un juge d'instruction comme Albanel et du " courage " qu'il a d'écrire que "l'école est le grand moyen de préservation de l'enfant ", car " on avait presque réussi on le sait, à ériger en 
dogme l'assertion contraire ${ }^{\left({ }^{(80)}\right.}$ Pourtant, si Fouillée reste à distance de ce débat, il n’en dénonce pas moins un système scolaire dominé par une conception intellectualiste et donc inapte à " relever la moralité publique ». ${ }^{(81)}$

Les conceptions qui émergent en ce début du XIXème siècle n'auront, pour certaines, guère de postérité. La vision intégrée s'effacera au profit d'une vision plus dualiste de l'esprit et du corps. Paradoxalement, la discipline c'est l'oubli de l'activité corporelle, c'est le silence intimé au corps, c'est la rigidité et l'uniformité des postures. Le procès que Dewey adressera à ce système d'éducation nous semble tout à fait pertinent pour rendre compte de ce système d'enseignement français. "Les enfants, prétendument consciencieux, consacrent ce qu'ils ont d'énergie à la tâche négative de réprimer leurs instincts et leurs tendances actives "; l'éducation consacre non l'utilisation des capacités corporelles, mais le « devoir strict de leur empêcher de leur donner libre cours ". ${ }^{(82)}$ L'éducation de la volonté, telle qu'elle se déploie alors, privilégie l'activité mentale. Octave Gréard, en 1889, cite L'éducation homicide de Victor de Laprade : "À ce milieu physique où le corps s'étiole, correspond un milieu moral non moins énervant pour le développement du caractère : une règle sèche, inflexible, qui brise les esprits et les courbe sous le joug d'une discipline formelle, qui, enveloppant l'enfant comme dans un réseau d'interdictions, anéantit tout à la fois sa volonté et l'irrite. " ${ }^{(83)}$ Vingt ans plus tard, Gréard constate que rien n'a changé dans le secondaire et dans l'université française. Mais, paradoxalement, l'école primaire échappe pour partie à cet oubli du corps. L'arrêté de juillet 1882 met au programme le travail manuel pour les garçons. Par « ces exercices du corps", il faut " prédisposer, en quelque sorte, les garçons aux futurs travaux de l'ouvrier et du soldat ».

\section{3. "Où iront-ils ?"}

\section{"Au crime, à la prison si vous les abandonnez"}

"A nos territoires lointains pour les féconder par le travail ; aux frontières de nos colonies pour les défendre souvent en héros si vous leur tendez à temps une main secourable... " ${ }^{(84)}$

L'organisation de l'éducation physique de l'école élémentaire - du moins si l'on ne se réfere qu'aux textes - semble en fait assez proche de cette éducation physique et morale du quartier correctionnel de Lyon, telle que Raux ${ }^{(85)}$ 
(86) M. Raux, Nos jeunes détenus. Études sur l'enfance coupable, Paris, G. Masson, 1890, p. 56.

(87) Ibid., p. 96.

(88) Ferdinand

Brunetière, "Éducation et instruction ", Revue des deux mondes, tome 127 , p. 914-934, p. 918.

(89) Ibid., p. 97.

(90) Ibid., p. 97.

(91) Ibid., p. 99. nous la dépeint à la même époque, en apparence tout au moins. Le régime appliqué aux jeunes détenus, de même que l'évaluation des résultats sont " considérés au quadruple point de vue physique, intellectuel, moral et professionnel $»{ }^{\left({ }^{(8)}\right)}$ Les journées comportent trois heures de récréation qui peuvent être agrémentées de jeux divers, sept heures de travail professionnel l'hiver et sept heures et demi l'été, ainsi que deux heures d'école. Ils se baignent une fois par semaine dans le Rhône et un professeur de gymnastique vient donner la leçon tous les dimanches. Outre les exercices aux agrès, la leçon revêt un caractère militaire et les exercices sont collectifs : flexions, " marches et mouvements cadencés " - au son du clairon -, " manœuvres avec fusil »... Cela n'est pas sans rappeler l'épisode des bataillons scolaires chers à Paul Bert, et par ailleurs cette orientation est revendiquée, puisque la "récompense suprême » - " très enviée » - est l'engagement dans l'armée. ${ }^{(87)}$ Une échelle des sanctions et des récompenses participe à définir l'éducation morale. Les méthodes doivent cependant composer avec un nouvel impératif pédagogique : "Former un citoyen ou former un soldat - je dis bien les former, et non pas les dresser -, c'est leur apprendre l'art de subordonner quelque chose d'eux-mêmes et de leurs droits naturels aux intérêts de la communauté. " ${ }^{(88)}$ C'est ce que préconisait le comte de Laborde et c'est ce qu'essaie de faire Raux dans le quartier correctionnel de Lyon, quand il nous décrit l'éducation morale des pupilles. À ce propos, Raux déplore, à double titre, que ces jeunes détenus frappés par application de l'art. 67 du Code pénal se voient interdire l'engagement volontaire et n'aient d'autre choix que la légion étrangère. ${ }^{\left({ }^{(9)}\right.}$ Il le regrette, puisque cela affaiblit son système de récompenses, et il déplore " qu'on les ait assimilés à des condamnés adultes ", ${ }^{(90)}$ ce qui participe aussi de cette difficulté à penser l'enfance dans sa différence.

Bien sûr, cette présentation ne doit pas faire oublier que les jeunes détenus de cet établissement modèle, âgés de 6 à 16 ans, sont parfois rebelles et qu' " il était d'une absolue nécessité de donner aux fonctionnaires chargés de les diriger un pouvoir considérable, une liberté d'action presque complète ${ }^{(91)}$

En fait, l'orientation donnée à cette éducation correctionnelle entretient une proximité assez étroite avec l'orientation donnée à l'école élémentaire. Léducation corporelle, l'éducation morale et le travail manuel sont les auxi- 
liaires d'une éducation ciblée qui doit substituer à l'impulsion native des motivations sociales conformes à la destinée sociale.

Dans ce XIXème siècle, les enfants des classes pauvres, quelles que soient leurs prédispositions, sont potentiellement une menace pour les biens, les personnes, la société ou la race. Qu'ils soient perçus comme incurables ou comme éducables, que la criminalité juvénile soit considérée comme atavique ou comme fait social, que le jeune criminel s'apparente à un "sauvage " ou à un " déchet de la civilisation ", peut certes ouvrir sur des perspectives différentes, mais dans tous les cas, ces dernières ne peuvent se penser qu'à l'intérieur d'une communauté de destin, qu'à l'aune d'une organisation sociale qui n'autorise que peu la mobilité sociale. L'éducation doit rappeler l'enfant des classes ouvrières aux devoirs et aux carrières vers lesquels l'engagent l'hérédité et le jeu naturel des forces sociales. 